\title{
Evaluación de la sostenibilidad económica del turismo de cruceros. Una aproximación metodológica en el Litoral Mediterráneo español
}

\section{Evaluation of cruise tourism economic sustainability. A methodological approach within the Spanish Mediterranean littoral}

Dr. Antonio Peláez Verdet es professor/investigador de la Facultad de Turismo de la Universidad de Málaga (España) (apv@ uma.es) (https://orcid.org/0000-0003-2545-5776).

Pilar Loscertales Sánchez es doctoranda en el Programa de Doctorado en Economía y Empresa de la Universidad de Málaga (España) (pilar.loscertales@uma.es) (https://orcid.org/0000-0002-2828-4338).

\begin{abstract}
Resumen
El turismo de cruceros es un poderoso dinamizador del turismo en los destinos en los que opera, y ha sido largamente estudiado desde el punto de vista del comportamiento del consumidor. También las dimensiones de la sostenibilidad medioambiental y social de este tipo de turismo han sido analizadas con detenimiento por la literatura. No obstante, y a pesar de un creciente número de investigaciones relacionadas con su impacto económico, no se ha llegado aún a una conclusión robusta sobre el efecto económico neto que tiene sobre los destinos. En este trabajo se estudia el impacto económico del turismo de cruceros en cuatro destinos del mediterráneo español con características distintas, usando datos de EGATUR y datos financieros de las empresas turísticas localizadas en los distintos destinos. Se correlacionaron, aplicando regresiones curvilíneas multivariantes, el tráfico crucerista en dichos destinos con la rentabilidad económica de las empresas bajo su área de influencia, así como su rentabilidad financiera y el número de empleados. Se establecieron, de esta manera, los umbrales en los que se estima que el turismo de cruceros tiene un efecto más beneficioso para la rentabilidad económica de las empresas turísticas que directamente se benefician del tráfico crucerista en destino, sin encontrarse relación con la rentabilidad financiera. También se describe la correlación del movimiento de pasajeros de crucero con el número de empleados declarado por las empresas.
\end{abstract}

\begin{abstract}
Cruise tourism is a powerful development agent on the destinations where it is present. It has been largely studied from the customer behaviour point of view. Moreover, its environmental and social sustainability dimensions have been deeply analysed by the literature. However, in spite of a growing number of researches related to its economic impact, no robust conclusion about its economic effect onto destinations has been reached so far. In this work we examine the economic impact of cruise tourism in four different Spanish Mediterranean destinations. For that we use data from EGATUR statistics and financial data from tourism companies in each destination considered. After running curvilinear multivariable techniques, we stablished a correlation between the cruise traffic and the economic profitability of those enterprises within its scope. Moreover, despite we found no significant correlation with finantial profitability, we also found evidence of significant connection between cruise traffic and the employment level of touristic firms affected.
\end{abstract}

\section{Palabras clave I keywords}

Turismo de cruceros, impacto económico, técnicas multivariables, litoral español, Mediterráneo, desempeño financiero Cruise tourism, economic impact, multivariate techniques, Spanish littoral, Mediterranean, Financial performance.

Forma sugerida de citar: Peláez Verdet, A., \& Loscertales Sánchez, P. (2018). Evaluación de la sostenibilidad económica del turismo de cruceros. Una aproximación metodológica en el Litoral Mediterráneo español. Retos Revista de Ciencias de la Administración y Economía, 15(8), 101-115. https://doi.org/10.17163/ret.n15.2018.07 


\section{Introducción y estado de la cuestión}

El turismo de cruceros, es dentro del sector turístico uno de los segmentos que mayor crecimiento ha presentado en los últimos años. Pese a la situación económica que ha tenido lugar en la última década, se ha contabilizado un incremento de la demanda global de cruceros de aproximadamente el 62\% según la Cruise Lines International Association (CLIA, 2016), posicionándolo como un producto en fase de crecimiento, siendo necesaria la realización de estudios que, desde una perspectiva cuantitativa, permitan conocer la importancia de esta actividad turística en el desarrollo económico en las regiones donde tiene lugar.

Una revisión de la literatura sobre el turismo de cruceros desde un enfoque económico pone de manifiesto los escasos estudios sobre la materia, centrándose, en la mayoría de los casos, en la definición del perfil crucerista y en las tendencias de esta tipología turística en el medio y largo plazo.

Por otro lado, profundizando en los estudios que abordan el impacto económico de los cruceros, afloran rápidamente tres focos de discusión: El análisis de las implicaciones económicas de la responsabilidad social corporativa llevadas a cabo por las compañías de cruceros, el estudio de los patrones de gasto relacionado con el turismo de cruceros, y la confirmación estadística para conocer el efecto económico de este tipo de actividad turística en una determinada región.

Profundizando en la literatura científica existente desde un punto de vista económico, se observa que los primeros estudios datan de mediados de la década de los ochenta, donde se pusieron de manifiesto las modelos input-output para conocer el impacto en las áreas donde se apreciaba una actividad turística. A este respecto Mescon \& Vozikis (1985) aplicaron esta metodología al condado de Miami Dade. Casi una década más tarde, Dwyer \& Forsyth (1996, 1998), basando sus estudios en áreas australianas de alta actividad crucerista, proponen otras metodologías alternativas al modelo input-output para conocer el impacto económico de los cruceros turísticos en cualquier región. Por otro lado, Vina \& Ford (1998) determinaron la importancia económica de una región en base a la clasificación entre los puertos de embarque y los puertos de recogida. Como hito que unificó los estudios que se habían llevado a cabo en los años noventa, Chase (2001), en su tesis, estableció la base para las investigaciones posteriores para conocer la influencia económica del turismo de cruceros en una región con una visión más amplia de las magnitudes evaluadas.

Los estudios posteriores continuaron utilizando la metodología input-output, y aunque hubo alguno que abordó el ámbito turístico crucerista a escala global (Wood, 2000), la mayoría analizó los efectos en una región particular, como fueron las investigaciones que realizaron Braun, Xander \& White (2002) en Port Canaveral; Chase \& Alon (2002) en Barbados y; Chase \& McKee (2003) en Jamaica. En este sentido, hay que destacar, que los resultados obtenidos en cada uno de ellos no confluían en conclusiones similares, debido ello al origen de los datos, ya que unos utilizaban encuestas y otros datos de contabilidad nacional.

Paralelamente Dwyer, Douglas \& Livaic (2004) continuaron profundizando en las regiones australianas, poniendo de manifiesto estar más enfocados en la reputación internacional de su industria de cruceros que por la imagen local de la misma. 
Los estudios más recientes, datados en la última década, incorporan como novedad la ampliación del ámbito geográfico, ya que se llevaron estudios centrados en el Caribe colombiano (v.gr. Brida, Bukstein, Garrido, Tealde \& Aguirre, 2010) y en las regiones de mediterráneas de Croacia y Serbia (v.gr. Dragin, Jovicic \& Boskovic, 2010). Asimismo se comienzan a medir magnitudes que en los estudios previos no se habían contemplado, como es el caso de Brida et al. (2010) que valoraron la capacidad de gasto de los pasajeros, algo que Dragin et al. (2010) no solo confirmaron, sino que enriquecieron, incorporando variables socioculturales al patrón de gasto.

Asimismo, Dragin et al. destacaron que los ingresos ocasionados por el turismo de crucero eran ventajosos para la industria turística local y para la creación de empleo, aunque no repercutían en los servicios de alojamiento y restauración, aunque otro equipo (Hefner, Mcleod \& Crotts, 2014), posteriormente, rebatiría en su estudio.

A partir de 2010 son muchos los estudios que se centran en el comportamiento del consumidor en los cruceros, destacando los realizados por Larsen et al. (2013) y por Hung \& Petrick (2010), quienes otorgan una escala válida y fiable para medir las restricciones de los consumidores en la adquisición de este tipo de viajes. Este aspecto ha sido considerado intensamente en los últimos años, en virtud de estudios como los de Fan \& Hsu (2014), que modelizaron el proceso de adquisición de los cruceristas, algo que posteriormente fue matizado por Chen et al. (2016). El comportamiento en puerto también ha sido investigado, llegándose a interesantes conclusiones en torno a la importancia de las recomendaciones (Satta et al., 2015) y otras fuentes de información que los turistas utilizan (Sanz-Blas et al., 2017).

Otras de las áreas de investigación, en referencia al turismo de cruceros, que más estudios ha generado, es el impacto medioambiental o social en las áreas receptoras de este tipo de turismo. A este respecto Kido-Cruz \& Cuellar-Río (2010) establecen la necesidad de crear un impuesto sobre el uso medioambiental en México a los pasajeros de los cruceros, determinando hasta el umbral necesario para que no afecte a la demanda turística. Por su parte, los estudios realizados en 2012 por Arnold et al. (2012), Brida et al. (2012) y Brida, Riaño \& Zapata (2012) en Alaska y Colombia respectivamente, revelan los procesos socioculturales que subyacen a la visita de los cruceristas. Los resultados obtenidos fueron afianzados por los estudios que se llevaron a cabo en el Mediterráneo (Brida, Chiappa, Meleddu \& Pulina, 2014).

Los estudios del sector realizados en los últimos cinco años, raramente tratan el impacto económico del turismo de cruceros, siendo uno de los escasos ejemplos el llevado a cabo en 2017 (Loscertales-Sánchez \& Peláez-Verdet, 2017), donde se aventuraban algunas medidas de impacto económico. Por el contrario, las líneas de investigación se han centrado en la responsabilidad social corporativa de las navieras (v.gr. Bonilla-Priego, Fontb \& Pacheco-Olivares, 2014; de Grosbois, 2015), el diseño de las instalaciones para satisfacer las demandas de compañías y usuarios (v.gr. Hardwick, Youdale \& Frankland, 2013), o las tendencias del mercado y la toma de decisiones en la gestión de destinos que ofrecen este tipo de servicio (v.gr. Castillo-Manzano, LópezValpuesta \& Alanís, 2014; De los Reyes, Ruiz, Ruiz \& De la Cruz, 2015). 


\section{Materiales y método}

Para esta investigación se han establecido dos etapas claramente diferenciadas. En la primera se han determinado los grupos en los que se subdividen los cruceristas en España, atendiendo a su comportamiento durante el viaje, enfocando el análisis especialmente en su gasto y en cuáles son las comunidades donde los cruceristas hacen turismo en España. Ello ha permitido identificar la importancia del litoral continental español en el mercado turístico nacional, acotándose los destinos receptores de turismo crucerista en esta área. Se logró así identificar cuatro destinos de distintas dimensiones candidatos a ser objeto de este estudio.

En una segunda fase se obtuvo información sobre las empresas que normalmente tienen vinculación con este sector, y que a priori podría verse afectada por una mayor o menor presencia de cruceros en el destino. La información financiera encontrada permitió establecer unas funciones de regresión que, convenientemente ajustadas, permitieron definir correlaciones entre la afluencia de pasajeros de crucero en cada destino y el rendimiento financiero de las empresas que operan en él.

\subsection{Identificación del perfil crucerista y su importancia en España}

La encuesta EGATUR es una campaña de prospección del mercado turístico que cada año se publica en España. Para esta investigación se necesitó, en primer lugar, recopilar los microdatos de la encuesta, disponibles en el Instituto Nacional de Estadística. Posteriormente hubo que consolidarlos y preprocesarlos en un software de Data Mining, que en este caso fue Weka (versión 3.8.1). El registro de estos microdatos permitió obtener un dataset de las siguientes variables y dimensiones:

Tabla 1. Variables de identificación del perfil del crucerista

\begin{tabular}{|l|l|l|}
\hline \multicolumn{1}{|c|}{ Variable } & \multicolumn{1}{|c|}{ Valores } & \multicolumn{1}{|c|}{$\begin{array}{c}\text { Frecuencia } \\
\text { (o Descripción) }\end{array}$} \\
\hline Fecha & Meses & $\begin{array}{l}\text { Entre octubre de } \\
2015 \text { y octubre de } \\
2017\end{array}$ \\
\hline Encuestado & $\begin{array}{l}\text { 2: Turista no residente (no tránsito) } \\
\text { 8: Turista no residente en tránsito }\end{array}$ & $\begin{array}{l}177826 \\
9741\end{array}$ \\
\hline Vía de salida & $\begin{array}{l}\text { 1: carretera } \\
\text { 2: aeropuerto } \\
\text { 3: puerto } \\
\text { 4: tren }\end{array}$ & $\begin{array}{l}22758 \\
154594 \\
7377 \\
2828\end{array}$ \\
\hline País de residencia & $\begin{array}{l}\text { 01: Alemania. 02: Bélgica. 03: Francia. 04: Irlanda. } \\
\text { 05: Italia. 06: Países Bajos. 07: Portugal. 08: Reino } \\
\text { Unido. 09: Suiza. 10: Rusia. 11: Países Nórdicos (Di- } \\
\text { namarca, Finlandia, Noruega, Suecia). 12: Resto de } \\
\text { Europa. 13: EEUU. 14: Resto de América. 15: Resto } \\
\text { del mundo }\end{array}$ \\
\hline
\end{tabular}




\begin{tabular}{|l|l|l|}
\hline $\begin{array}{l}\text { Comunidad au- } \\
\text { tónoma principal } \\
\text { del viaje }\end{array}$ & $\begin{array}{l}\text { 01: Andalucía. 02: Aragón. 03: Principado de Astu- } \\
\text { rias. 04: Illes Balears. 05: Canarias. 06: Cantabria. } \\
\text { taluña. 10: Comunitat Valenciana. 11: Extremadura. } \\
\text { 12: Galicia. 13: Comunidad de Madrid. 14: Región } \\
\text { de Murcia. 15: Comunidad Foral de Navarra. 16: } \\
\text { País Vasco. 17: La Rioja. 18: Ceuta. 19: Melilla }\end{array}$ & \\
\hline $\begin{array}{l}\text { Total pernoctacio- } \\
\text { nes }\end{array}$ & Noches & $\begin{array}{l}\text { Media: 9,27 } \\
\text { Desv. Std: 12,9 }\end{array}$ \\
\hline Alojamiento & $\begin{array}{l}\text { 1: Hoteles y similares } \\
\text { 2: Resto de mercado } \\
\text { 3: Alojamiento no de mercado }\end{array}$ & $\begin{array}{l}105466 \\
21581 \\
60520\end{array}$ \\
\hline Motivo del viaje & $\begin{array}{l}\text { 1: Ocio/vacaciones } \\
\text { 2: Negocios } \\
\text { 3: Resto }\end{array}$ & $\begin{array}{l}134254 \\
18830 \\
34483\end{array}$ \\
\hline $\begin{array}{l}\text { Usa paquete turís- } \\
\text { tico }\end{array}$ & $\begin{array}{l}\text { 1: Sí } \\
\text { 6: No }\end{array}$ & $\begin{array}{l}38269 \\
149298\end{array}$ \\
\hline $\begin{array}{l}\text { Gasto total duran- } \\
\text { te el viaje }\end{array}$ & Euros & $\begin{array}{l}\text { Media: } 1132,69 \\
\text { Desv. Std: 1040,6 }\end{array}$ \\
\hline
\end{tabular}

Tras la obtención de estos datos, un análisis cluster de K Medias aplicado únicamente a las unidades de observación con vía de salida 3 (puerto) permitió identificar los principales cinco grupos que componen el sector de cruceristas en España, con interesantes resultados. Básicamente pudo identificarse la rotunda importancia que tienen las comunidades de Cataluña, Levante y Andalucía como lugar vacacional de los turistas que posteriormente salen vía marítima. Esto, a su vez, arrojó luz sobre los principales puertos que usan estos turistas, lo que dio paso a una segunda fase de esta primera etapa de la investigación, buscando acotarla geográficamente.

Figura 1. Pasajeros en los principales puertos españoles

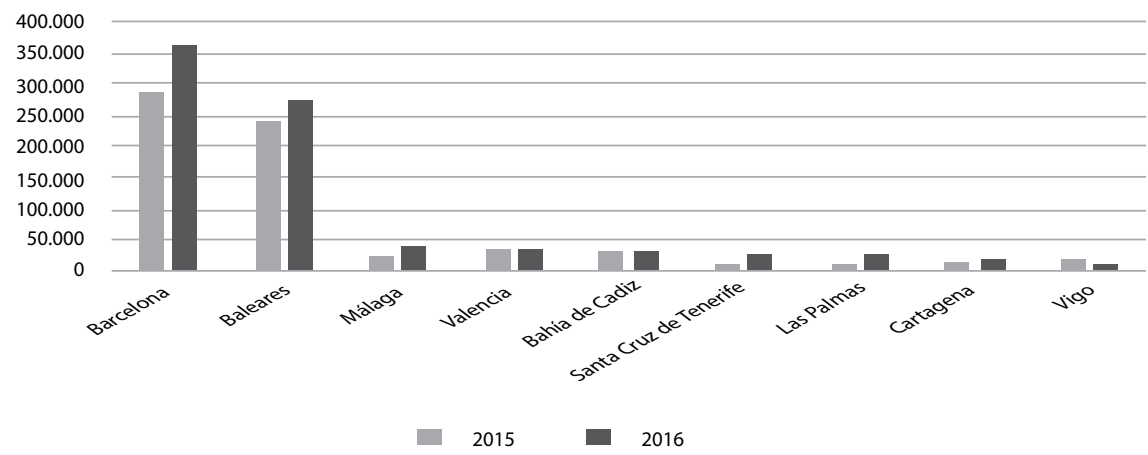


En este sentido, los analistas acudieron al Anuario Estadístico de Puertos del Estado, editado cada año por Puertos del Estado (dependiente del Ministerio de Fomento). Este manual presentó para la temporada alta de cruceros (que coincide con el mes de julio) de los años 2015 y 2016 las cifras que pueden verse en la figura 1, que presentan la situación para los puertos españoles que mantuvieron en esos años un tráfico superior a 10000 pasajeros. Como puede apreciarse, existen cuatro puertos del litoral español peninsular que presentan dimensiones distintas, muy atractivos para el análisis: Barcelona (puerto líder en cruceros), Málaga y Valencia (puertos intermedios), y Cartagena (puerto de dimensión más reducida).

Este escenario proporcionó la oportunidad de efectuar un análisis comparativo del impacto económico que producen los cruceros en cada uno de estos destinos, que podía medirse mediante el rendimiento económico de las empresas más vinculadas con este sector.

\subsection{Rendimiento financiero de las empresas}

No todas las empresas de cada destino turístico están afectadas de igual manera por la industria de los cruceros. Para los propósitos de esta investigación, se obtuvieron medidas de la rentabilidad económica y financiera de una serie de empresas en las plazas de Barcelona, Málaga, Valencia y Cartagena, mediante la base de datos SABI (Sistema de Análisis de Balances Ibéricos). Los criterios de selección de las empresas tenían que cumplir el objetivo de conseguir un conjunto de firmas que fueran sensibles a la variación con el tiempo del número de pasajeros que desembarcan en cada uno de los puertos analizados. En este sentido, la investigación se centró en conseguir la información financiera de las compañías de cada cuidad que cumplieran simultáneamente los siguientes requisitos:

Tabla 2. Requisitos de selección de empresas objeto de estudio

\begin{tabular}{|c|c|}
\hline Requisito & Valor \\
\hline Creada & Desde el 1 de enero de 1997 \\
\hline Estatus & Activa durante 2017 \\
\hline $\begin{array}{l}\text { CNAE } 2009 \\
\text { (Código pri- } \\
\text { mario) }\end{array}$ & $\begin{array}{l}471 \text { - Comercio al por menor en establecimientos no especializados } \\
472 \text { - Comercio al por menor de productos alimenticios, bebidas y tabaco en } \\
\text { establecimientos especializados } \\
476 \text { - Comercio al por menor de artículos culturales y recreativos en estableci- } \\
\text { mientos especializados } \\
551 \text { - Hoteles y alojamientos similares } \\
552 \text { - Alojamientos turísticos y otros alojamientos de corta estancia } \\
561 \text { - Restaurantes y puestos de comidas } \\
563 \text { - Establecimientos de bebidas } \\
791 \text { - Actividades de agencias de viajes y operadores turísticos } \\
799 \text { - Otros servicios de reservas y actividades relacionadas con los mismos } \\
900 \text { - Actividades de creación, artísticas y espectáculos } \\
910 \text { - Actividades de bibliotecas, archivos, museos y otras actividades culturales } \\
920 \text { - Actividades de juegos de azar y apuestas } \\
932 \text { - Actividades recreativas y de entretenimiento }\end{array}$ \\
\hline
\end{tabular}


El resultado de esta búsqueda arrojó un total de 5640 empresas (3 163 en Barcelona, 199 en Cartagena, 825 en Málaga y 1453 en Valencia). Sus medidas de rentabilidad económica, rentabilidad empresarial y su número de empleados entre 1997 y 2016 fueron incorporados al análisis.

Por otra parte, al consultarse el Anuario Estadístico del Registro de Buques y Empresas Navieras (proporcionado por la Dirección General de la Marina Mercante, Secretaría de Estado de Infraestructuras, Transporte y Vivienda, Ministerio de Fomento), se obtiene el censo de pasajeros registrado por cada autoridad portuaria, con un horizonte temporal de veinte años. Esto permitió a los investigadores disponer de una variable independiente que correlacionar con la dependiente del desempeño financiero de las empresas que rodean cada puerto de cruceros. Este proceso se llevó a cabo mediante un proceso de estimación curvilínea que ajustó la relación entre ambas variables, mediante el concurso del software IBM SPSS Statistics® (versión 22; IBM Corp., 2013).

\section{Análisis}

Como consecuencia de la aplicación de estos métodos, el análisis pudo fundamentar una medida de la influencia cruzada del turismo de cruceros en cuatro destinos españoles del mediterráneo peninsular. Para ello hizo falta, en primer lugar, identificar al cliente crucerista y estimar los puertos en los que estaba presente de manera turística, y en segundo lugar calcular la correlación entre la afluencia de pasajeros de crucero en cada puerto con el rendimiento económico de las empresas asociadas a la misma.

\section{1. ¿Qué destinos visitan los cruceristas antes de regresar?}

Tras la discretización de la variable «Gasto total» en diez intervalos de igual amplitud, y el filtrado de las encuestas para obtener solo los turistas de crucero (y no los excursionistas o aquellos turistas que hubieran salido por otros medios), el número total de turistas por crucero quedó en 3 873). La ejecución del análisis clúster en el dataset obtenido de EGATUR entre octubre de 2015 y octubre de 2017 y preprocesado así se pudo establecer, usando un algoritmo de K Medias (Distancia Euclídea) un número de tres grupos distintos de cruceristas que integraban el total de turistas, que se distribuyeron como sigue:

Tabla 3. Resultados del análisis cluster sobre datos EGATUR

\begin{tabular}{|l|l|l|l|}
\hline \multicolumn{1}{|c|}{ Clúster } & \multicolumn{1}{c|}{$\mathbf{1}$} & \multicolumn{1}{c|}{$\mathbf{2}$} \\
\hline Origen & Reino Unido & Reino Unido & Resto del Mundo \\
\hline $\begin{array}{l}\text { Comunidad Autónoma de destino } \\
\text { principal }\end{array}$ & Cataluña & $\begin{array}{l}\text { Comunidad } \\
\text { Valenciana }\end{array}$ & Andalucía \\
\hline Total pernoctaciones (promedio) & 7,42 & 38,14 & 11,8 \\
\hline Motivo principal de viaje & Vacaciones & Vacaciones & Vacaciones \\
\hline Paquete Turístico & No & No & No \\
\hline Gasto total en el viaje & $\begin{array}{l}\text { Entre } 1450 \text { y } \\
1990 \text { euros }\end{array}$ & $\begin{array}{l}\text { Más de 1990,60 } \\
\text { euros }\end{array}$ & $\begin{array}{l}\text { Más de 1990,60 } \\
\text { euros }\end{array}$ \\
\hline
\end{tabular}

De este análisis pueden colegirse dos conclusiones preliminares: el turista de cruceros generalmente soporta un nivel de gasto elevado en comparación con otros 
tipos de turista, hecho apoyado por que los centroides de los tres clúster se sitúan en el escalón más alto de las categorías de gasto general (caso de clúster 2 y 3), y el inmediatamente inferior (clúster 1). Además, ello permitió a los investigadores acotar geográficamente el análisis en el litoral peninsular, ya que en ningún momento parece que los puertos insulares representen un peso específico comparado con los destinos de Cataluña, Comunidad Valenciana y Andalucía.

Comparadas las conclusiones parciales anteriores con el informe de Puertos del Estado referido anteriormente, el análisis prosiguió centrándose en los puertos litorales peninsulares del Mediterráneo: Barcelona (Cataluña), Valencia (Comunitat Valenciana), Cartagena (Puerto asociado al destino Valencia por proximidad geográfica, e interesante desde el punto de vista de su dimensión) y Málaga (puerto crucerista de entrada a Andalucía desde el Mediterráneo).

\section{2. ¿Qué influencia tiene el turismo de cruceros en las empresas locales?}

En una segunda fase de la investigación, el equipo centró sus esfuerzos en recopilar información financiera sobre las empresas que más influencia podrían soportar, a priori, en el sector de los cruceros. Tras la búsqueda descrita en el segundo punto del epígrafe anterior, el equipo registró y promedió anualmente las rentabilidades económicas y financieras, así como el número de empleados declarado de las 5640 empresas involucradas en este estudio de las que se tenía conocimiento en la base SABI a través del Registro Mercantil español. Tras esta labor de recopilación, se llegó a disponer de un histórico, empresa a empresa, de las variables que se describen seguidamente:

Tabla 4. Descriptivos de la rentabilidad económica de las empresas por ciudad analizada

\begin{tabular}{|l|r|r|r|r|}
\multicolumn{1}{c|}{$\begin{array}{c}\text { Rentabilidad } \\
\text { Económica (\%) }\end{array}$} & Barcelona & Cartagena & \multicolumn{1}{c|}{ Málaga } & Valencia \\
\hline Media & $-4,28$ & 1,91 & $-7,27$ & $-5,33$ \\
\hline Error típico & 9,39 & 1,00 & 2,37 & 1,31 \\
\hline Mediana & $-7,58$ & 1,50 & $-3,41$ & $-5,73$ \\
\hline Desviación estándar & 42,00 & 4,45 & 10,60 & 5,87 \\
\hline Varianza & $1.763,68$ & 19,84 & 112,42 & 34,43 \\
\hline Curtosis & 14,35 & $-0,96$ & 1,88 & $-1,09$ \\
\hline Coeficiente de asimetría & 3,32 & $-0,04$ & $-1,53$ & $-0,05$ \\
\hline Rango & 231,80 & 14,83 & 39,54 & 20,25 \\
\hline Mínimo & $-70,57$ & $-5,18$ & $-35,49$ & $-15,49$ \\
\hline Máximo & 161,22 & 9,65 & 4,05 & 4,76 \\
\hline Nivel de confianza (95,0\%) & 19,65 & 2,08 & 4,96 & 2,75 \\
\hline
\end{tabular}

La rentabilidad económica es la medida de la eficiencia con la que la empresa arroja resultados, en función del tamaño de su activo. Se entiende como el cociente entre el beneficio antes de intereses e impuestos y el total activo comprometido para obtener esos beneficios en un año concreto. En este sentido, cabe destacar que las empresas de 
Cartagena exhiben mayor prosperidad que las de las otras tres ciudades, que muestran un promedio muy inferior de rentabilidades económicas en el periodo considerado.

Tabla 5. Descriptivos de la rentabilidad financiera de las empresas analizadas, por ciudad

\begin{tabular}{|l|r|r|r|r|}
\hline \multicolumn{1}{c|}{$\begin{array}{c}\text { Rentabilidad } \\
\text { Financiera (\%) }\end{array}$} & Barcelona & Cartagena & \multicolumn{1}{c|}{ Málaga } & Valencia \\
\hline Media & 20,08 & $-53,56$ & 72,20 & 32,64 \\
\hline Error típico & 34,12 & 36,12 & 66,72 & 25,61 \\
\hline Mediana & 8,20 & 24,01 & 33,77 & 23,55 \\
\hline Desviación estándar & 152,61 & 161,53 & 298,38 & 114,55 \\
\hline Varianza & $23.289,56$ & $26.092,20$ & $89.032,20$ & $13.121,13$ \\
\hline Curtosis & 7,53 & 4,44 & 3,52 & 5,81 \\
\hline Coeficiente de asimetría & 2,14 & $-2,16$ & 0,32 & 1,37 \\
\hline Rango & 772,72 & 645,82 & $1.547,28$ & 612,10 \\
\hline Mínimo & $-225,28$ & $-548,91$ & $-682,19$ & $-213,41$ \\
\hline Máximo & 547,44 & 96,91 & 865,09 & 398,69 \\
\hline Nivel de confianza (95,0\%) & 71,42 & 75,60 & 139,65 & 53,61 \\
\hline
\end{tabular}

La rentabilidad financiera, por su parte, expresa la medida en la que la empresa retribuye a sus accionistas la inversión que han efectuado en ella, mediante la generación de los recursos de capital previstos. Debe entenderse como la proporción de los beneficios antes de intereses e impuestos y los recursos propios de la empresa. En este caso puede decirse que las empresas malagueñas son las que mejor retribuyen los recursos con que sus accionistas las dotan.

Tabla 6. Descriptivos del número de empleados declarado por las empresas estudiadas en cada ciudad

\begin{tabular}{|l|r|r|r|r|}
\hline \multicolumn{1}{|c|}{ Empleados } & Barcelona & Cartagena & Málaga & Valencia \\
\hline Media & 12,03 & 6,94 & 7,24 & 7,91 \\
\hline Error típico & 0,47 & 0,50 & 0,44 & 0,39 \\
\hline Mediana & 12,15 & 6,71 & 7,71 & 7,69 \\
\hline Desviación estándar & 2,09 & 2,19 & 1,98 & 1,72 \\
\hline Varianza & 4,36 & 4,78 & 3,92 & 2,95 \\
\hline Curtosis & 1,66 & $-0,65$ & 1,59 & 2,62 \\
\hline Coeficiente de asimetría & $-1,07$ & $-0,15$ & $-1,32$ & 0,74 \\
\hline Rango & 8,78 & 7,88 & 7,95 & 8,32 \\
\hline Mínimo & 6,50 & 2,67 & 2,00 & 4,20 \\
\hline Máximo & 15,28 & 10,55 & 9,95 & 12,52 \\
\hline Nivel de confianza (95,0\%) & 0,98 & 1,05 & 0,93 & 0,83 \\
\hline
\end{tabular}


Los empleados son una medida estándar de la dimensión de una empresa. Es fácil deducir, a través de los datos reflejados en la tabla 6, que las firmas barcelonesas son las que mayor dimensión tienen en términos de puestos de trabajo mantenidos en su estructura. Por el contrario, las empresas del resto de plazas observadas mantienen unas medias de empleados en torno a seis, lo que permite suponer que son de menor entidad que las firmas de Barcelona.

Estas son medidas asociadas a la vida económica de los últimos veinte años de las empresas analizadas en cada destino. Por otra parte, el objetivo era ponerlas en correlación con sus respectivos tráficos de pasajeros, variable que se obtuvo del Registro de Buques de la Marina Mercante, que publica oficialmente esta medida para cada puerto español. Esta medida da una indicación de la importancia turística de cada plaza desde el punto de vista turístico, y permite comparar los destinos con una magnitud homogénea y fácil de comprender.

Tras la recopilación de la información proporcionada por el Registro de Buques de la Marina Mercante, se obtuvo un registro completo no solo de pasajeros, sino de actividad de cada puerto en relación con tráfico de naves, carga, servicios, cabotaje, etc. A los efectos de esta investigación, la información relevante sobre los pasajeros fue automatizada en un registro único conteniendo el tráfico de pasajeros durante los mismos veinte años analizados para cada puerto, que puede verse en la tabla siguiente:

Tabla 7. Descriptivos del flujo de pasajeros por puerto analizado

\begin{tabular}{|l|r|r|r|r|}
\hline \multicolumn{1}{|c|}{ Pasajeros } & \multicolumn{1}{c|}{ Barcelona } & \multicolumn{1}{c|}{ Cartagena } & \multicolumn{1}{c|}{ Málaga } & \multicolumn{1}{c|}{ Valencia } \\
\hline Media & $2.586 .072,60$ & $59.566,90$ & $552.781,20$ & $471.231,60$ \\
\hline Error típico & $231.287,47$ & $12.644,92$ & $55.046,81$ & $50.002,03$ \\
\hline Mediana & $2.709 .430,50$ & $32.187,50$ & $556.612,00$ & $397.458,50$ \\
\hline Desviación estándar & $1.034 .349,02$ & $56.549,79$ & $246.176,84$ & $223.615,88$ \\
\hline Varianza & $1.069 .877 .900 .044,78$ & $3.197 .879 .046,52$ & $60.603 .034 .507,85$ & $50.004 .061 .213,09$ \\
\hline Curtosis & $-1,33$ & $-0,26$ & $-0,91$ & $-0,97$ \\
\hline Coeficiente de asimetría & $-0,36$ & 0,97 & 0,13 & 0,66 \\
\hline Rango & $3.073 .589,00$ & $186.111,00$ & $800.326,00$ & $702.263,00$ \\
\hline Mínimo & $887.840,00$ & $2.401,00$ & $184.712,00$ & $205.880,00$ \\
\hline Máximo & $3.961 .429,00$ & $188.512,00$ & $985.038,00$ & $908.143,00$ \\
\hline $\begin{array}{l}\text { Nivel de confianza } \\
(95,0 \%)\end{array}$ & $484.090,24$ & $26.466,12$ & $115.214,31$ & $104.655,45$ \\
\hline
\end{tabular}

Es relevante mencionar que la tendencia en el volumen de pasajeros ha sido creciente en todo el periodo considerado, lo que obliga a pensar en una mejoría sostenida de las condiciones para las empresas. Esto se pone de manifiesto en la figura 2. 
Figura 2. Pasajeros por puerto analizado y año

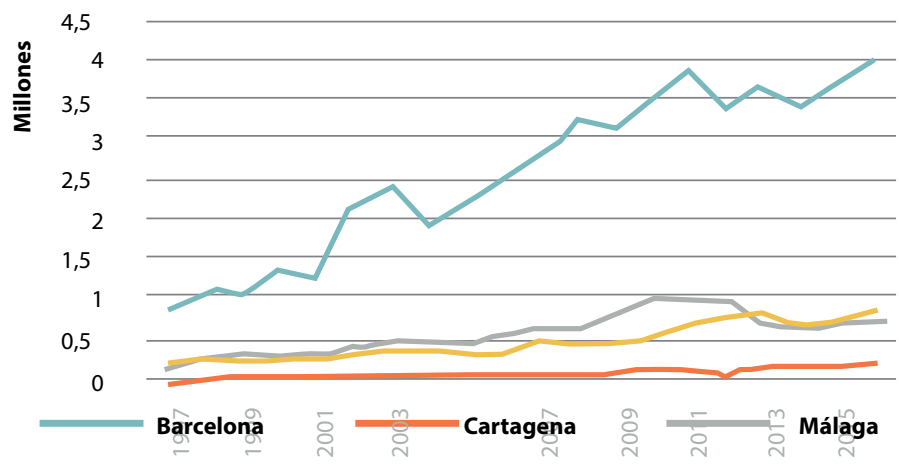

Valencia

\section{Resultados}

Del cruce de estos conjuntos de datos se pudieron obtener conclusiones interesantes, una vez introducido todo en el software SPSS ${ }^{\circledR}$ y efectuando una correlación curvilínea para estimar qué curvas se ajustarían mejor al comportamiento mutuo de los parámetros descritos. Los resultados pueden apreciarse en la tabla 8 (las celdas en blanco equivalen a relaciones no significativas):

Tabla 8. Funciones y coeficientes significativos al 95\% del ajuste

Rentabilidad Económica-Pasajeros por ciudad

\begin{tabular}{|c|c|c|c|c|}
\hline \multirow{2}{*}{\multicolumn{2}{|c|}{$\begin{array}{l}\text { Modelo de ajuste } \\
\text { Cartagena }\end{array}$}} & \multicolumn{3}{|c|}{ Pasajeros/Rentabilidad Económica } \\
\hline & & \multirow{2}{*}{$\begin{array}{l}\text { Málaga } \\
\text { Coef. }\end{array}$} & \multirow{2}{*}{ Valencia } & \multirow[b]{2}{*}{ Coef. } \\
\hline Función & Parámetro & & & \\
\hline \multirow{2}{*}{ Inverso } & 1/Pasajeros & & 4004608,24 & 3552515,8 \\
\hline & Constante & & $-16,545$ & $-14,715$ \\
\hline \multirow{2}{*}{ Lineal } & Pasajeros & $-5,19 \mathrm{E}-05$ & & \\
\hline & Constante & 5,292 & & \\
\hline \multirow{2}{*}{ Logarítmico } & $\ln ($ Pasajeros $)$ & $-2,993$ & $-11,69$ & $-8,749$ \\
\hline & Constante & 33,719 & 145,991 & 107,842 \\
\hline \multirow{3}{*}{ Cuadrático } & Pasajeros & 0 & & \\
\hline & Pasajeros**2 & $5,20 \mathrm{E}-10$ & & \\
\hline & (Constante) & 7,27 & & \\
\hline \multirow{4}{*}{ Cúbico } & Pasajeros & & 0 & \\
\hline & Pasajeros $* * 2$ & & $-7,59 \mathrm{E}-10$ & \\
\hline & Pasajeros $* * 3$ & & $4,42 \mathrm{E}-16$ & \\
\hline & Constante & & $-47,043(*)$ & \\
\hline
\end{tabular}

Nota: (*) significativo al $90 \%$. 
Como puede apreciarse, los resultados indican relaciones significativas en varias funciones, pudiendo destacarse que algunas de ellas se revelan con una alta significación. No se obtuvieron resultados significativos para el mismo análisis asociado a la rentabilidad financiera de las empresas analizadas, lo que indica una ausencia de correlación entre ésta y el flujo de pasajeros, pudiendo estar relacionada con otras magnitudes económicas o turísticas.

En cuanto a los puestos de trabajo que declaran estas empresas, se encontraron relevantes correlaciones, puestas de manifiesto en la tabla 9:

Tabla 9. Funciones y coeficientes significativos al $95 \%$ del ajuste entre flujo de pasajeros y número de empleados declarado por puerto

\begin{tabular}{|c|c|c|c|c|}
\hline \multirow{2}{*}{\multicolumn{2}{|c|}{$\begin{array}{l}\text { Modelo de ajuste } \\
\text { Barcelona }\end{array}$}} & \multicolumn{3}{|c|}{ Pasajeros/Número de empleados } \\
\hline & & \multirow{2}{*}{$\begin{array}{l}\text { Cartagena } \\
\text { Coef. }\end{array}$} & \multirow{2}{*}{$\begin{array}{l}\text { Málaga } \\
\text { Coef. }\end{array}$} & \multirow[b]{2}{*}{ Coef. } \\
\hline Función & Parámetro & & & \\
\hline \multirow{2}{*}{ Inverso } & 1/Pasajeros & $-3025668,14$ & $-18970,137$ & $-1095660,37$ \\
\hline & Constante & 13,485 & 7,768 & 9,779 \\
\hline \multirow{2}{*}{ Lineal } & Pasajeros & & & $4,33 \mathrm{E}-06$ \\
\hline & Constante & & & 4,844 \\
\hline \multirow{2}{*}{ Exponencial } & Pasajeros & & & $8,75 \mathrm{E}-07$ \\
\hline & Constante & & & 4,229 \\
\hline \multirow{2}{*}{ Logarítmico } & $\ln ($ Pasajeros $)$ & & & 2,511 \\
\hline & Constante & & & $-25,682$ \\
\hline \multirow{3}{*}{ Cuadrático } & Pasajeros & & $6,05 \mathrm{E}-05(*)$ & \\
\hline & Pasajeros**2 & & $-3,02 \mathrm{E}-10$ & \\
\hline & (Constante) & & 5,247 & \\
\hline \multirow{4}{*}{ Cúbico } & Pasajeros & $4,32 \mathrm{E}-05$ & 0 & \\
\hline & Pasajeros**2 & $-1,77 \mathrm{E}-11$ & $-3,23 \mathrm{E}-09$ & \\
\hline & Pasajeros**3 & $2,23 \mathrm{E}-18$ & $1,03 \mathrm{E}-14$ & \\
\hline & Constante & $-18,554$ & 2,156 & \\
\hline \multirow{2}{*}{ S } & 1/Pasajeros & $-354853,492$ & $-3235,46$ & $-223281,54$ \\
\hline & Constante & 2,641 & 2,023 & 2,443 \\
\hline
\end{tabular}

Nota: (*) significativo al $90 \%$. 


\section{Discusión y conclusiones}

De los resultados presentados anteriormente se extraen interesantes conclusiones que podrían servir de referencia para orientar futuros trabajos sobre este aspecto del turismo crucerista.

Lo primero que llama la atención es el número de funciones que correlacionan significativamente el volumen de pasajeros que circuló en cada puerto con las rentabilidades económicas registradas de las empresas analizadas. En el caso de Málaga las funciones logarítmica o cúbica parecen responder bastante bien, mientras que en caso de Cartagena una función cúbica ajusta de manera aceptable esta relación.

Es llamativa la ausencia de relaciones entre la rentabilidad financiera de las empresas y el número de cruceristas. Podría aventurarse que tiene que ver con la estructura de capital y el endeudamiento de las firmas que se han analizado. En futuros trabajos ésta debería ser una línea de investigación, que profundice en los pasivos de las empresas estudiadas y verifique si existen categorías de empresas financieramente más susceptibles que otras a los cambios en el mercado de cruceros de su ciudad.

El número de puestos de trabajo parece ser una variable incluso más vinculada al flujo de cruceros que la propia rentabilidad económica de las empresas en las que sirven. Es llamativo el caso de Málaga, donde una función compuesta explica muy bien esta relación. No obstante, una función logística de parámetros parecidos en todas las ciudades podría destacarse como un hallazgo relevante, ya que permite explicar de manera significativa y muy parecida las relaciones entre los puestos de trabajo y el flujo de turistas en los cuatro destinos analizados, pudiendo deducirse por sus coeficientes el impacto que tendría un aumento del flujo de turistas en la creación de puestos de trabajo.

Finalmente, podrían apuntarse algunas líneas de acción para futuros trabajos en este sentido, que indaguen en lo ya presentado aquí. Por ejemplo, una de las limitaciones de este trabajo ha sido el horizonte temporal que se ha considerado. En la medida que se puedan ir agregando datos temporales a las series estudiadas, para hacerlas más largas únicamente veinte años, los resultados deberían ir ganando precisión. Por otra parte, el perfil de las empresas no ha sido disgregado en el análisis, pudiendo haber empresas muy sensibles al sector y otras categorías a los que cambios en el flujo turísticos de los cruceros les perjudique o beneficie menos. Un análisis más pormenorizado sobre cómo afectan estas alteraciones dependiendo, por ejemplo, del epígrafe de la CNAE 2009 en la que se encuentre la empresa podría arrojar resultados muy prometedores y profesionalmente valiosos para los DMO's de estos cuatro destinos estudiados.

\section{Referencias}

Arnold, A., Arnold, B., \& Chapman, D. (2012). Alaskan Communities Responses Regarding the Economic, Sociocultural and Enviromental Impact of the Cruise Ship Tourism Industry. Insight to a Changing World Journal, (2), 29-42.

Bonilla-Priego, M. J., Fontb, X., \& Pacheco-Olivares, M. del R. (2014). Corporate sustainability reporting index and baseline data for the cruise industry. Tourism Management, 44, 149160. https://doi.org/10.1016/j.tourman.2014.03.004

Braun, B. M., Xander, J. A., \& White, K. R. (2002). The impact of the cruise industry on a region's economy: A case study of Port Canaveral, Florida. Tourism Economics, 8(3), 281-288. https://doi.org/10.5367/000000002101298124 
Brida, J. G., Bukstein, D., Garrido, N., Tealde, E., \& Aguirre, S. Z. (2010). Impactos económicos del turismo de cruceros: Un análisis del gasto de los pasajeros de cruceros que visitan el Caribe colombiano. Estudios y Perspectivas en Turismo, 19, 607-634.

Brida, J. G., Chiappa, G. del, Meleddu, M., \& Pulina, M. (2014). A Comparison of Residents' Perceptions in Two Cruise Ports in the Mediterranean Sea. International Journal of Tourism Research, (16), 180-190. https://doi.org/10.1002/jtr

Brida, J. G., Del Chiappa, G., Meleddu, M., \& Pulina, M. (2012). Cruise tourism externalities and residents' support: A mixed approach. Economics, 6(40), 0-27. https://doi.org/10.5018/ economics-ejournal.ja.2012-40

Brida, J. G., Riaño, E., \& Zapata, S. (2012). Residents' perceptions toward cruise tourism impacts on a community: a factor and cluster analysis. Cuadernos de Turismo, (29), 263-266.

Castillo-Manzano, J. I., López-Valpuesta, L., \& Alanís, F. J. (2014). Tourism managers' view of the economic impact of cruise traffic: the case of southern Spain. Current Issues in Tourism, 18(7), 701-705. https://doi.org/10.1080/13683500.2014.907776

Chase, G., \& Alon, I. (2002). Evaluating the Economic Impact of Cruise Tourism: A Case Study of Barbados. Anatolia, 13(1), 5-18. http://dx.doi.org/10.1080/13032917.2002.9687011

Chase, G. L. (2001). The economic impact of cruise ships in the 1990s: Some evidence from the Caribbean. ProQuest Dissertations and Theses. Kent State University. (goo.gl/6CNcGX) (8-11-2017).

Chase, G., \& McKee, D. (2003). The economic impact of cruise tourism on Jamaica. Journal of Tourism Studies, 14(2), 16-22.

Chen, J. M., Neuts, B., Nijkamp, P., \& Liu, J. (2016). Demand determinants of cruise tourists in competitive markets: Motivation, preference and intention. Tourism Economics, 22(2), 227-253. https://doi.org/10.5367/te.2016.0546

CLIA (2016). 2017 Cruise Industry Outlook. (goo.gl/CRi3fm) (8-11-2017).

de Grosbois, D. (2015). Corporate social responsibility reporting in the cruise tourism industry: a performance evaluation using a new institutional theory based model. Journal of Sustainable Tourism, 9582 (May), 1-25. https://doi.org/10.1080/09669582.2015.1076827

De los Reyes, E., Ruiz, C., Ruiz, E., \& De la Cruz, R. (2015). Tendencias y estrategias en la industria del crucero: principales áreas del turismo de cruceros en el mundo. TURyDES, 8(19).

Dragin, A. S., Jovicic, D., \& Boskovic, D. (2010). Economic Impact of Cruise Tourism Along the Pan-European Corridor Vii. Ekonomska Istrazivanja, 23(4), 127-141. https://doi.org/10.1 080/1331677X.2010.11517438

Dwyer, L., Douglas, N., \& Livaic, Z. (2004). Estimating the Economic Contribution of a Cruise Ship Visit. Tourism in Marine Environments, 1(1), 5-16.

Dwyer, L., \& Forsyth, P. (1996). Economic Impacts of Cruise Tourism in Australia. Journal of Tourism Studies, 7(2), 36-43.

Dwyer, L., \& Forsyth, P. (1998). Economic significance of cruise tourism. Annals of Tourism Research, 25(2), 393-415. https://doi.org/10.1016/S0160-7383(97)00098-4

Hardwick, L., Youdale, J., \& Frankland, R. (2013). Cruise ship driven development of port and coastal infrastructure. Coasts and Ports 2013 (pp. 352-357). National Committee on Applied Mechanics. (goo.gl/DWFBWB) (12-10-2017).

Fan, D. X. F., \& Hsu, C. H. C. (2014). Potential Mainland Chinese Cruise Travelers' Expectations, Motivations, and Intentions. Journal of Travel and Tourism Marketing, 31(4). https://doi. org/10.1080/10548408.2014.883948

Hefner, F., Mcleod, B., \& Crotts, J. (2014). Research note: An analysis of cruise ship impact on local hotel demand - An event study in Charleston, South Carolina. Tourism Economics, 20(5), 1145-1153. https://doi.org/10.5367/te.2013.0328

Hung, K., \& Petrick, J. F. (2010). Developing a Measurement Scale for Constraints To Cruising. Annals of Tourism Research, 37(1), 206-228. https://doi.org/10.1016/j.annals.2009.09.002

IBM Corp. (2013). IBM SPSS Statistics for Windows [Computer software]. Armonk, NY: IBM Corp. 
Kido-Cruz, M. T., \& Cuellar-Río, M. (2010). Impacto económico por el pago de pasajeros de cruceros para la conservación de recursos naturales: el caso de Bahías de Huatulco, México. Economía Sociedad y Territorio, $x(32), 81-97$.

Loscertales-Sánchez, P., \& Peláez-Verdet, A. (2017). The economic influence of cruise tourism onto a littoral destination. International Journal of Scientific Management Tourism, 3(2), 351-367. (https://goo.gl/uyH71J) (8-10-2017).

Mescon, T. S., \& Vozikis, G. S. (1985). The economic impact of tourism at the port of Miami. Annals of Tourism Research, 12(4), 515-528. https://doi.org/10.1016/0160-7383(85)90075-1

Satta, G., Parola, F., Penco, L., \& Persico, L. (2015). Word of mouth and satisfaction in cruise port destinations. Tourism Geographies, 17(1), 54-75. https://doi.org/10.1080/14616688. 2014.938689

Sanz-Blas, S., Buzova, D., \& Carvajal-Trujillo, E. (2017). Investigating the moderating effect of information sources on cruise tourist behaviour in a port of call. Current Issues in Tourism 20(2), 120-128. https://doi.org/10.1080/13683500.2015.1091444

Vina, L. de la, \& Ford, J. (1998). Economic Impact of Proposed Cruiseship Business. Annals of Tourism Research, 25(4), 205-208.

Wood, R. E. (2000). Caribbean cruise tourism: Globalization at sea. Annals of Tourism Research, 27(2), 345-370. https://doi.org/10.1016/S0160-7383(99)00073-0 